\title{
New measurement method corrected a failure in special relativity
}

\section{Viktor Schatz ( $\nabla$ v.schatz@yahoo.de )}

Private person https://orcid.org/0000-0002-6700-5776

\section{Article}

Keywords: special relativity, Lorentz transformation, particle physics

Posted Date: June 7th, 2021

DOI: https://doi.org/10.21203/rs.3.rs-583131/v1

License: (c) (i) This work is licensed under a Creative Commons Attribution 4.0 International License. Read Full License 


\section{New measurement method corrected a failure in special relativity}

V.Schatz

A logical reasoning problem in the Special Relativity interpretation of the Lorentz transformation was discovered and corrected by introducing an alternative interpretation of Lorentz transformations with a relativistic measuring process based on both, stretched time and length units, whereby the measurements as a consequence measure a shortened distance and time lines as variables due to used units. The space shuttles are getting longer instead of shorter and a barn-pole paradox does not occur. The spatial locations remain in their locations and space is not contracted and not changed. The failure is that by contracting both units and distance the Lorentz factor is eliminated and relativity was vanished. The elementary particles have their own measuring devices for time and length in the form of wavelengths and frequencies and they read distances and durations of different lengths from space using their own relativistically different units. The new interpretation is covered by the two postulates of Einstein. This is a sensational result too in view of all dramatic and romantic history as there is really a failure discovered. The Lorentz transformations remain being in use with another method. The new theory and the discovered problems of logical failures are explained vividly in the same manner as Special Relativity was using easy be thought samples. Reader do not need a high level of mathematics to be fit for as it is a more qualitatively thematic for every one who is interested in relativity matters.

\section{Motivation}

Every one knows the relativistic literature romantics about time dilation and that is again a reason to look at.

The heated historical debate about Einstein's Special Relativity Theory, SRT, an interpretation of the Lorentz transformations was only conducted from the point of view of negating the relativity. Hence, no one thought of the third possibility, now discovered by chance - that the relativistic is real, but that Einstein, Lorentz and Poincare may have misinterpreted it. Or they could be more then one suitable interpretations too, which we prefer to think at start.

It was a sudden discover and solution at once of a really one failure in the SRT's interpretation of a measurement of the length, which is now changed using stretched relativistically length units in order to measure less units as a contracted again distance between two space locations.

Even if it is hart to believe after all this is true and anyone will himself understand it fully and vividly. 


\section{Discovery of an alternate interpretation of Lorentz Transformations besides SRT}

We think of the following thought experiment in space with several fast spaceships. A route is set up between space stations A and B with quasi "fixed space locations", but there are spaceships of different generations, each with a different speed, with Lorentz factor $\gamma_{1}, \gamma_{2}$ and $\gamma_{3}$.

Each of the 3 has read out a different distance $A B$ in the same space between the same locations in its own Inertial Frames, IF: $\mathrm{AB} / \gamma_{1}, \mathrm{AB} / \gamma_{2}$ and $\mathrm{AB} / \gamma_{3}$. It is completely the same and even to each of the 3 spacemen what the others had measured - they measure it independently of each other. $A B$ is measured in earthly dimensions. Because the faster ones arrive earlier, they wait for the ones to come later and it happens. Although it takes a longer distance, all three arrive at the same destination, brake there and get off. They all started from A and all ended up in the same localisation B. We of course do idealise this experiment as they always are to think of, because all cosmic bodies are in movement, but in a large scale of distances and high speeds we can approximately mean, that a location of a star planet system is likely to be in same space place. The later coming space ship will have to do some correction of his rout.

From this starting we can already see that places A and B in space must have remained the same. Spatial locations do not shift in order to adjust the traveller's routes relativistically. How can it be then? These spatial locations A and B, as ponderable masses of planets, are also events that are Lorentz invariant.

The only possibility is that everyone had a different measure of length with which they read this distance $\mathrm{AB}$ from space. Consequently, the measures of length, and also the measures of time, thought logically, must be adapted relativistically.

How can one count a fewer length units, of meters in the same space distance AB? Well, if the meter or any length unit gets longer. Stretched.

But the SRT tells us to contract all lengths including especially the length measure units too. So who is right? We know how the answer of physics is at this moment.

The time dilation means, as we continue, that the 3 travellers had a time-dilated heartbeat compared to earths IF. The period is increased, enlarged, dilated. If you send a signal to earth for every heartbeat you can mark the corresponding point in space. Between the 3 travellers there are distances of different lengths between heartbeats. We could also use an atomic clock as a pacemaker. The larger the Lorentz factor gamma, the slower the heartbeat and the longer a heartbeat space segment that can be used as a measure unit of length. So less of such longer heart-beat space segments will fit between A and B fit in the faster spaceships IF causing a shorter measured distance. We will lead the reader to that understanding about how particles periodic "heart beating" and moving is producing our time flow in dependence of the speed.

Who wants to shorten the reading of step by step details can just have a look at Fig. 2 and 3 and compare and understand all by himself. 


\section{Distance Measurement according to SRT and discovering that failure of a century}

In SRT there is a requirement to contract all lengths. This is a procedure and is used. It does not just follow from Lorentz transformations. An earthly known distance $\mathrm{AB}[\mathrm{m}]$ means that we know how often the earths meter between A and B fits.

Consequently, a distance measured in the Inertial Frame of a traveller would also be $\mathrm{A}^{\prime} \mathrm{B}^{\prime}\left[m^{\prime}\right]$ in meters $m^{\prime}$ of the traveller, no longer in earthly meters $m$.

Now we follow the SRT and contract and see that it has stayed within meters of earths $m$. Then where are IF's own relativistic meters $m$ ?

$$
\mathrm{A}^{\prime} \mathrm{B}^{\prime}=\mathrm{AB}[m] / \gamma
$$

This is to see in Fig. 1 b) after applying the SRT contraction method to the previous situation in a).. And also SRT says that the on-board meter also contracts to:

$$
m^{\prime}=m / \gamma
$$

Shifted

$$
m=m^{\prime} \gamma \text {. }
$$

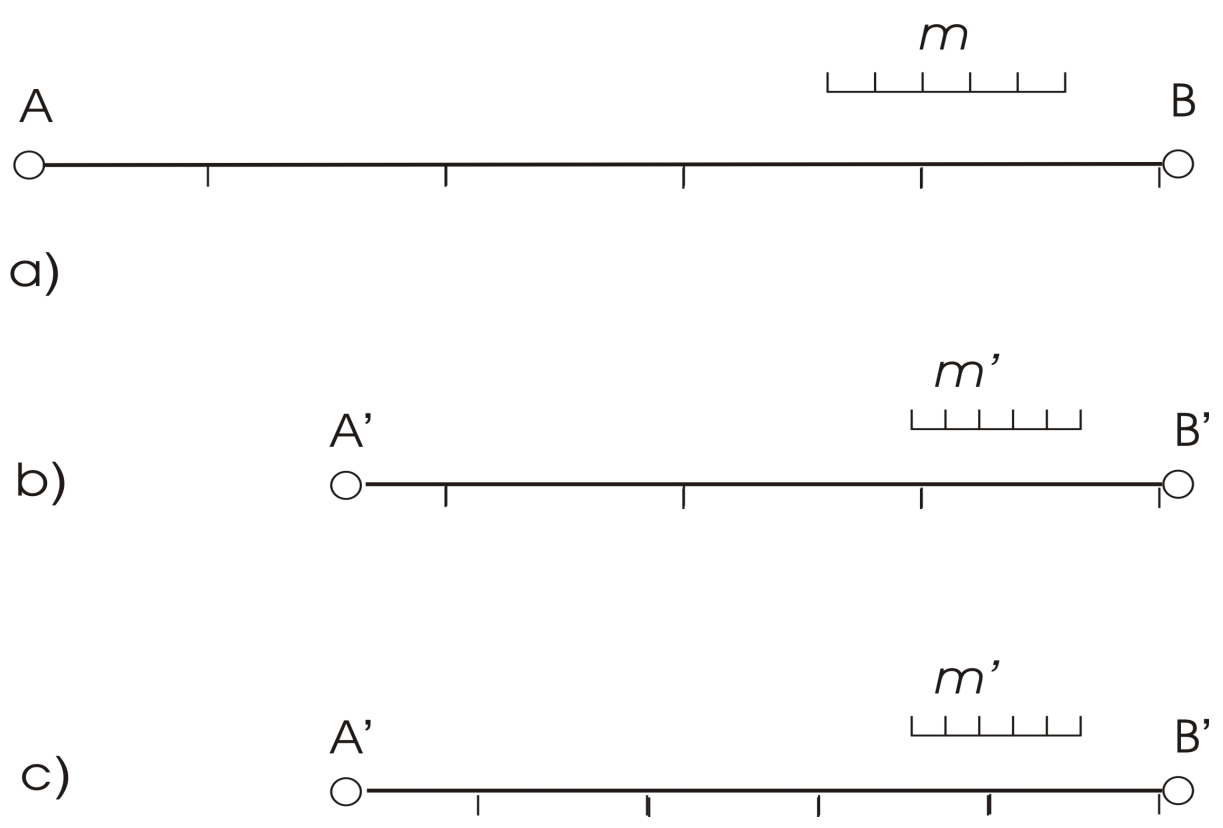

Fig.1 Distance Measurement according to SRT in b), and the avoided step in c). with the failure

And we think we have to somehow get the relativistic meter into (1) properly so that our measuring method is correct again. We use. 


$$
\mathrm{A}^{\prime} \mathrm{B}^{\prime}=\mathrm{AB}\left[m^{\prime} \gamma\right] / \gamma
$$

Because gamma is a constant factor for the current IFs, we can take it from the unit.

$$
\mathrm{A}^{\prime} \mathrm{B}^{\prime}=\gamma \mathrm{AB}\left[m^{\prime}\right] / \gamma=\mathrm{AB}\left[m^{\prime}\right]
$$

And get that $\mathrm{A}^{\prime} \mathrm{B}^{\prime}$ is the same as $\mathrm{AB}$, but measured with new meters $m^{\prime}$. We have already given up his gamma, both gammas are abbreviated. What is there now? If we now think unnoticed, we measure the $\mathrm{AB}$ with $m^{\prime}$ and get the result. AB hasn't changed, but $m^{\prime}$ is contracted?

In the case when it is thought contracted again, we get more short meters $\mathrm{m}^{\prime}$ between $\mathrm{A}=\mathrm{A}^{\prime}$ and $\mathrm{B}=\mathrm{B}^{\prime}$, so the distance increase instead of contracting. Or in the case, if we leave it equal to earthly meter $\mathrm{m}$, we get back the earthly distance.

The logic is that the correct result of the contracted distance from (1) comes about through a wrong procedure, in which one has measured with earthly meters. Then you go there and also choose the meter. But one also forgot to use it and thus kept the relativity completely switched off. The two gammas cancel out.

In Fig. 1 b) we see it graphically in a more didactic way that the $\mathrm{A}^{\prime} \mathrm{B}^{\prime}$ were contracted, bur the old units tell as the viewer relativistic number of units fitting between $\mathrm{A}^{\prime}$ and $\mathrm{B}^{\prime}$. And besides that independently the length unit was contracted, but not used.

In Fig. 1 c) we see that avoided step of the method of SRT. We have to drop old unit $m$ and instead to use the new contracted unit $m^{\prime}$ to find out how many times it will fit between $A^{\prime}$ and $B^{\prime}$. And voila we get again old number of units fitting between $\mathrm{A}^{\prime}$ and $\mathrm{B}^{\prime}$ which we see in Fig. 1 a) before starting that method.

The "number of units fitting between" is the "rate of distance to the length unit", but the distance here is meant before a measurement as an unknown distance. We don't have a word for that yet. Maybe to call it an "absolute distance", gap or a route? We cannot assign any numbers to that abstract unmeasured route gap, which is expecting a measurement, because that would be already after a measurement possible only. And the length measurement is connected to the IF by its relativistic own length unit.

A recalibration of the distance between two IF equals a process if we recalculate from $\mathrm{km}$ to miles.

\section{Muon case study}

In Fig. 2 we see a typical image of the relativistic case of atmospherically Muons as can be found in many standard literature courses [1] teaching Relativity of SRT.

We have only marked the start and end points of the Muon route with A and B and the mountain peak $\mathrm{C}$ in space. As you can see, these points were shown offset in both Inertial Frames. For 
undisclosed reasons and motivation, the lower end point $\mathrm{B}$ was retained and only the upper point $\mathrm{A}$ and $C^{\prime}$ moved in the space in order to contract the entire space.

Are the points A and B no longer in the same spatial locations for all Inertial Frames? Do two events take place at two different locations A and A' instead of one? Any number of Inertial Frames can be derived with different Lorentz gamma - and then there should be an infinite number of new displaced locations of the mass particles and other "contraction centre locations"? Does it physically move the locations in space? If so, how it is with the mass of matter?

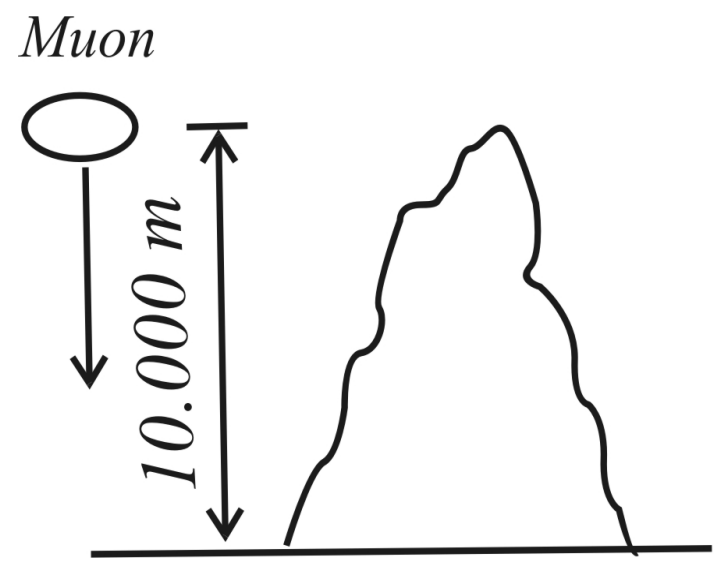

a)

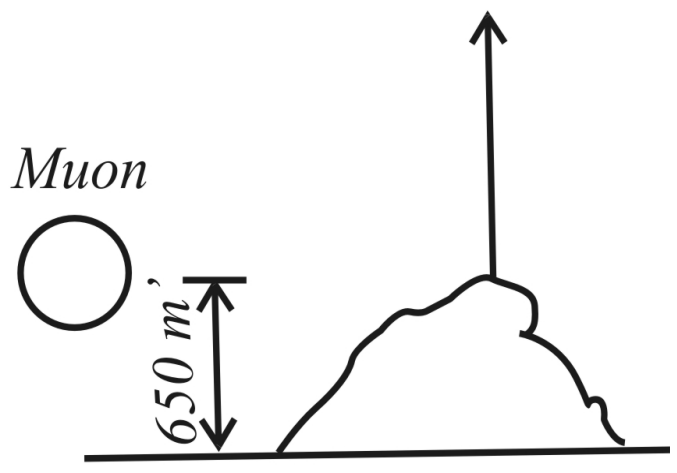

b)

Fig. 2. A common representation of the length contraction a Muon track in the atmosphere represented from an university standard teaching materials [1]: a) earths Inertial Frame, b) Muon Inertial Frame.

We refer to the discussion above about the same spatial locations that go nowhere. It is the traveller who actively do something and all travellers did meet in the same space location.

Projection or mapping of the distance AB to A'B' between the two Inertial Frames in Fig. 2 is produced by the method of SRT shortening (contracting) both the distance and the length unit. It means that the relativistically contracted distance A'B' is after this method just having $10.000 \mathrm{~km}$ ' again, only measured with shorted (contracted) length unit' the shorted distance'. It is like a rubber tape with length units marks on it, when after contracting it will keep the number of marks and the result of measurement will stay the same, eliminating the contraction at all.

The relation rate of the route length to the length unit resulting in a measured distance is to be changed relativistically to a viewer number, but according to SRT it was kept Lorentz invariant.

Each reader may use any one of standard literature on Special Relativity Theorie, the SRT [2] to get enough inside knowledge to be fit for this lecture.

\section{Muon and the new measurement of distance by stretching the unit of length and time}

A Muon lives longer and moves on with relativistically stretched measures of length and time. 
The Muons in the atmosphere are almost as fast as light, live and travel $10 \mathrm{~km}$ instead of $650 \mathrm{~m}$, which corresponds to a gamma-Lorentz factor of approximately 15.

In Figure 2 it can be clearly seen that the terrestrial observer sees the Muons lifespan $A B$ as $10 \mathrm{~km}$, while a spaceman travelling at the same speed with the Muon sees his Muon lifespan as his "own normal" A'B' with $650 \mathrm{~m}^{\prime}$. Consequently mapping in space $650 \mathrm{~m}^{\prime}$ between the same space locations $\mathrm{A}=\mathrm{A}^{\prime}$ and $\mathrm{B}=\mathrm{B}^{\prime}$ at the beginning and end of the route of the Muon to the $10 \mathrm{~km}$ distance seen from the earthling, so that the unit length of the Muon stretched in terms of the earth by Lorentz factor. Instead of to be contracted according to SRT.

As contracted, the Muon spaceman measures the distance AB instead of earthly $10 \mathrm{~km}$ as his own $650 m^{\prime}$ in relativistic Muon meters $m^{\prime}$, which apply in his Inertial Frame. 1 Muon meter $1 m^{\prime}=15 m$ in earth meters. With horizontal lines we have divided the distance $650 \mathrm{~m}^{\prime}$ to approx. $100 \mathrm{~m}^{\prime}$, which corresponds to $1500 \mathrm{~m}$ on the earthling side. As you can see, it is a projection of, a direct mapping the Muonic $650 \mathrm{~m}^{\prime}$ on the $10 \mathrm{~km}$ of the terrestrial spatial spatial distance AB. In a third IF, a third, relativistically measured distance measurement is obtained with a third straight relativistic length dimension.

The spaceship of the Muon spaceman is measured by him as normal with his own Muon's meters $m^{\prime}$ and consequently the spaceship is also elongated - not contracted! The distance AB can also be measured in spaceship lengths as an unit of length and only then after converted into Muon meters $m^{\prime}$. The space ship is itself an unit of length.

The $650 \mathrm{~m}$ ' Muon's lifespan for "normal length" results in the well-known examples if an almost light-speed of $98 \%$ is classically multiplied with the mean lifespan of $2.2 \mu \mathrm{s}$, as it is called in the well-known representations. However, since a "normal Muon" can be slower, this distance has to be even shorter and then the Lorentz gamma would be much larger. However, this does not change anything in the fundamental statement of the relativity. Some sources also mention a gamma of 50 .

From Figure 2 it can be seen directly that the Muons lifespan was stretched from the own $650 \mathrm{~m}$ compared to the Inertial Frame on earth to fit the $10 \mathrm{~km}$ of earths IF between the space points A and B. So not contracted! The measured distance, which an earthling sees as $10 \mathrm{~km}$, was "contracted" by evaluating or reading out the same stretch of space with longer length measurements units in the Muons Inertial Frame in the spaceship. So the wording "contraction" did play a game to all physicians for 115 years. That is, the words are influencing the thinking. To have many different languages may help it. And German is the best one for science as we see in the history of physics last two hundred years.

Let be a classical measurement of a distance between America and Europe in miles and in $\mathrm{km}$. The numbers figured due to different units are different, but the spaces "really distance" is same.

With time dilation we proceed in exactly the same way by stretching the time unit, which is obvious at constant speed and stretched length unit $\mathrm{t}=\mathrm{S} / \mathrm{V}$. In Muon-IF own Muon lifetime passes from the normal laboratory-measured average $\mathrm{T}^{\prime}=2.2 \mu \mathrm{s}^{\prime}$, which is proportional to the length of units can be divided along the line AB. And in the earth IF with the same Lorentz factor 15, more of the shorter time units must be read out, i.e. times gamma 15 to $33 \mu \mathrm{s}$. 
One can clearly see in the picture that the actual spatial locations A and B in the space remain the same in both compared IF. If we could, one can also perceive the same spatial locations from an nth IF, but reading the distance $A B=f(\gamma)$ with its own relativistic units of length in numbers differently than $(\mathrm{AB})$ ', $(\mathrm{AB})$ " etc..

That is a very important finding showing that the spatial locations and space remain the same, i.e. the space is not compressed or stretched. $\rightarrow$ The moving matter particles just "read" the space differently, relativistically with IF's own units of measurement for length and time (and mass). Therefore they have wave length and frequencies and this is what is changing relativistically on elementary level of matter. Our macroscopic clocks and meters and miles are composed of and mapped to the particles wave lengths and frequency's.

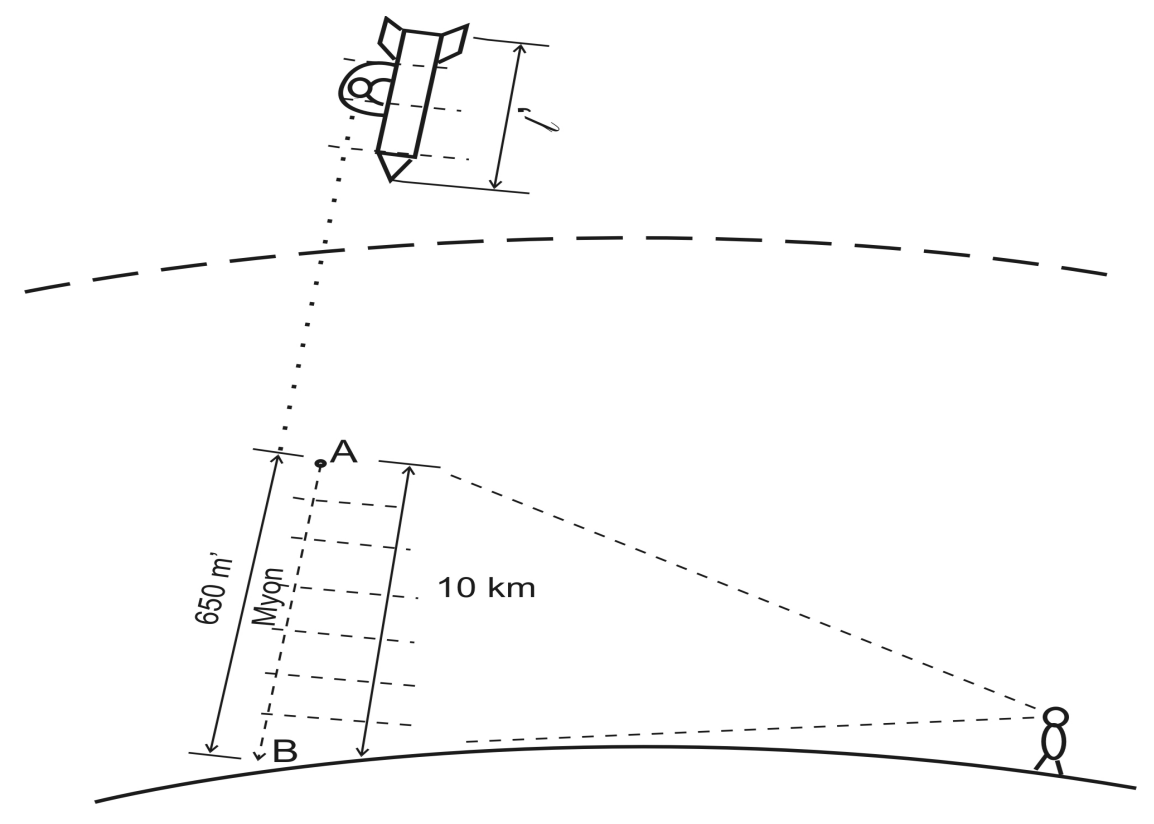

Fig. 3. A relativistic Muon observed in the earth's atmosphere from earths Inertial Frame.

We can also conclude, that the Muon was in points $\mathrm{A}=\mathrm{A}^{\prime}$ and $\mathrm{B}=\mathrm{B}^{\prime}$ simultaneously as an invariant event himself being there. How different the information travels different time to somewhere due to different distances and IF's is another question raising after that. In Fig. 2 one could think that this are 2 different points in space time with $\mathrm{A} \neq \mathrm{A}^{\prime}$ and $\mathrm{B} \neq \mathrm{B}^{\prime}$ and they are then two different realities about 2 Muons as events instead of one. We must contradict this interpretation of reality as invariant in Lorentz transformations. Fig. 3 explains at a single look how relativistic contracting distances works even if it was not the starting point of present discovery. The stretching of the moving objects is meant physically really of course.

\section{The new relativistic length of the space shuttle}

The length $l$ of the spaceship can be clearly read graphically in Figure 3: the lines with the units of length $m^{\prime}$ of the Muon's IF show that it has an intrinsic length of approx. $400 \mathrm{~m}^{\prime}$. Consequently it is also stretched and is measured stretched by earth people with gamma $\gamma=15$ to about $6000 \mathrm{~m}$ on earth. According to SRT, it should be measured to be $26.66 \mathrm{~m}$ contracted for earthly IF. The difference is a failure of squared Lorentz factor $\gamma^{2}$. 
Every relativistic spaceship has its own space length in its own IF, measured with IF's own length measure units. We assume that this unit is $100 \mathrm{~m}$ length in figure 3 . Because every meter is now relativistically stretched, from the relativistic point of view of the earth IF, the length of this ship is also stretched for the earths IF. Longer instead of shortened according to SRT. With Gamma 15 , the earthling sees the $100 \mathrm{~m}^{\prime}$ spaceship as approx. $1500 \mathrm{~m}$ long, measured with the earthly unit dimensions.

This new relativistic interpretation solution of Lorentz transformations applied to the meausre of lengths is unambiguously and equally unequivocally assessed asymmetrically from both IF. The spaceman in the "Muon spaceship" sees with his stretched meter $m^{\prime}$ unit measured all earthly objects in his direction of movement as contracted because less of his length dimensions fit inside. In opposite direction the earths observer will see the space ship stretched measuring it with his shorter length units.

So it is clearly assessed relativistic asymmetrically and the famous "barn-pole paradox" does not appear at all. And we got like for time dilation same asymmetry between compared IF's.

\section{New measurement method using a stretched length unit}

In accordance to Fig. 1 we can see the new method figured out.

In Fig.4 it is the new measurement process to see.

We use Lorentz factor as an units rate with $\gamma=m^{\prime} / m$ for stretching length unit according to new method and interpretation. Unit $m$ is here for meter.

$\mathrm{AB}=\mathrm{Nab} \cdot[m]$; with $\mathrm{Nab}$ being just the numerical value without unit $m$;

$\mathrm{A}^{\prime} \mathrm{B}^{\prime}=\mathrm{Na}^{\prime} \mathrm{b}^{\prime} \cdot\left[m^{\prime}\right]$;

$\mathrm{AB}=\mathrm{A}^{\prime} \mathrm{B}^{\prime}$

aus $(5) \rightarrow \mathrm{Na}^{\prime} \mathrm{b}^{\prime}=\mathrm{Nab} \cdot[m] /\left[m^{\prime}\right]=\mathrm{Nab} / \gamma ; \quad$ with $\mathrm{Na}^{\prime} \mathrm{b}^{\prime}<\mathrm{Nab}$

\section{$m$}

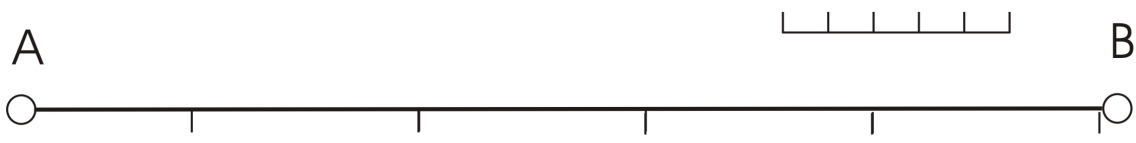

B

a)

$m^{\prime}$

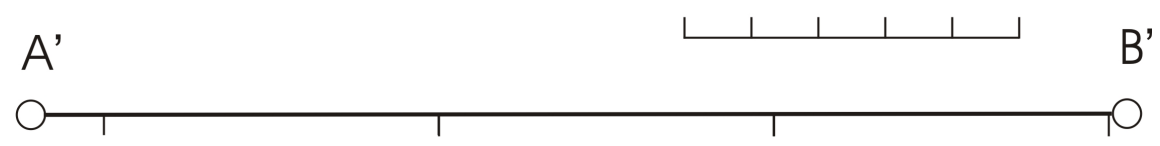

b)

Fig. 4 New measurement method using stretched length unit meter $m^{\prime}=\gamma m$. 
And that is a true result with a contracted distance as measured value.

$\rightarrow$ if using the contracted unit $m^{\prime}=m / \gamma$ it will lead to opposite result with $\mathrm{Na}^{\prime} \mathrm{b}^{\prime}>\mathrm{Nab}$, which contradicts SRT. And if the distance $\mathrm{A}^{\prime} \mathrm{B}^{\prime}=\mathrm{AB} / \gamma$ would be also contracted before that, it leads to $\mathrm{Na}^{\prime} \mathrm{b}^{\prime}=\mathrm{Nab}$, cancelling the relativistic contraction at all, and that is actually happening in SRT's interpretation as to see in Fig.1.

\section{Difference between units and distances}

What is the difference between an unit of length and a measured distance?

The measured distance is a product of the unit with a numerical value without unit, which is a counter of how many times the unit fits in between of any two points in space. So, the units we change only one time and then we measure with them an infinite number of distances within our new IF. This was the philosophical difficulty in the present case of relativistic theories. We have too much common use daily experience with units and distances and so we do not recognise how to divide it exactly. Some one will say it is an easy piece of thoughts, but history shows another side.

The units are relativistically to be stretched in moving IF, if transforming from a relativistically lower to a relativistically higher IF and vice versa. And distances are to measure after that with those relativistic changed units to find out the numerical scalar variable value of a measured distance. Even if the result is true when we just did directly contracted a distance, behind that is this measurement mechanic. A macroscopic sample model we have if we measure same distance with miles or km getting different variables.

The same is true for time units and measured time segments or lines, just it is a more abstract entity and is to handle using a speed of movement to connect distances with time. In our case the speed of movement is speed of light for photons and speed of observed IF's for moved mass particles.

\section{Without the "barn-pole paradox"}

We can see how the old good "barn pole paradox" is avoided in the present new interpretation of Lorentz transformation, explained in Fig. 5.

In Fig. 5 a) the earths observer can measure with his normal length meter $m$ and time sec. units, that the units of the space shuttle meter', sec.' are stretched longer and he see the shuttle therefore also stretched and clocks slower, which we figured out as oval shaped.

In Fig. 5 b) in opposite observation the space shuttle observer is measuring with his for him normal units, that the length meter and time units sec. on the earth are relativistically contracted and so are also the mass of the bar and the men himself. The vertical dimensions are kept as in SRT. This is the new view in the present interpretation of Lorentz transformations.

To show more clear we use larger and smaller sized letters for moved an stationary IF's just temporary to make sure we do not lose the path. 


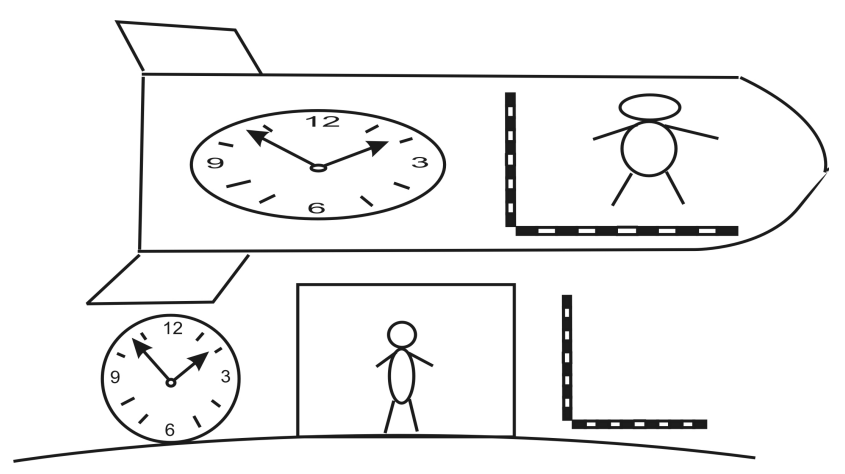

a)

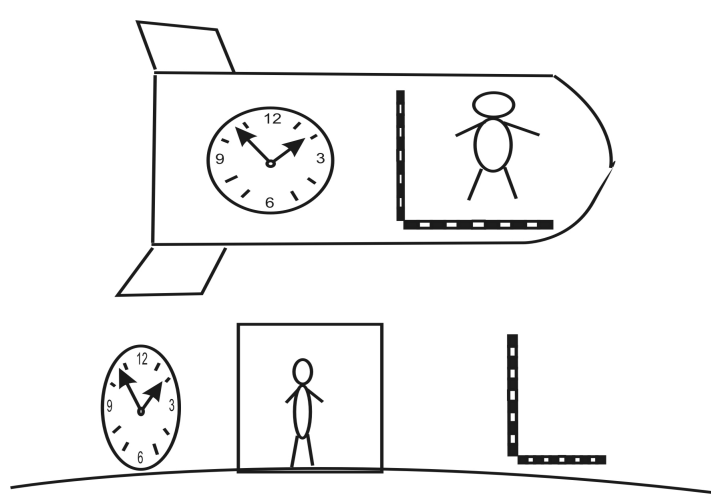

b)

Fig. 5. A new situation free of a "barn-pole paradox" a) in earths IF, b) in space IF both asymmetrically different.

We can write asymmetrically relations now for length and time units to be stretched with $\Delta$ standing for units:

$$
\Delta l=\Delta l \gamma ; \quad \text { or in old form } \Delta l^{\prime}=\Delta l \gamma ; \quad \Delta l^{\prime}>\Delta l ;
$$

in backwards compare direction reciprocally length unit

$$
\Delta l=\Delta l / \gamma ; \text { or in old form } \Delta l=\Delta l 1 \gamma ;
$$

The distance in space $\mathrm{AB}$ is as quasi "absolute" the same, so $\mathrm{AB}=\mathrm{A}^{\prime} \mathrm{B}^{\prime}$, just it will be differently measured. The measured distance in a value without units we name respective $\mathrm{n}$ and $\mathrm{N}$ or in old style $N^{\prime}$ and $N$. This is later identical to $l$ and $l^{\prime}$, just to take care we did it didactically.

$$
\begin{gathered}
\mathrm{AB}=n \cdot \Delta l=\mathrm{A}^{\prime} \mathrm{B}^{\prime}=N \cdot \Delta l \\
N=n \cdot \Delta l / \Delta l=n / \gamma=l^{\prime}=l / \gamma . l^{\prime}<l ;
\end{gathered}
$$

With $n$ the distance measured in earths IF and $N$ distance scalar value measured in shuttles IF contracted as required.

For time in same method of measurements using units or periodic time stretching $\Delta T$.

$$
\Delta T=\Delta T \gamma ; \quad \text { or in old form } \Delta T^{\prime}=\Delta T \gamma ; \Delta T^{\prime}>\Delta T
$$

in backwards direction reciprocally time dilation as in SRT

$$
\Delta T=\Delta T / \gamma ; \quad \text { or in old form } \Delta T=\Delta T^{\prime} / \gamma
$$

measuring an abstract "time space segment" between A and $\mathrm{B}(\mathrm{AB})_{\mathrm{t}}$ in analogy to distance

$$
(\mathrm{AB})_{\mathrm{t}}=t \cdot \Delta T=\left(\mathrm{A}^{\prime} \mathrm{B}^{\prime}\right)_{\mathrm{t}}=t^{\prime} \cdot \Delta T \text {; }
$$




$$
t^{\prime}=t \cdot \Delta T / \Delta T=t \cdot \Delta T / \Delta T^{\prime}=t / \gamma . \quad t^{\prime}<t
$$

Now this is didactically better written about the new measurement method in relativistic physics. In (14) one can see that in "moved clocks" was less time $t^{\prime}<t$ measured during the trip between A and $\mathrm{B}$. The time $t^{\prime}$ measured in a moved IF counts viewer scalar variable number of units then compared with static earths IF $t$ during a trip between A and B. This result is what SRT is verbally telling us if the traveller twin stays younger, but was never written that clear way. Einstein and followers didn't understand to differ units and measurements. It is a philosophical point of view about space as one continuum and all what is possible in continuum was to stretch or contact it as whole including units. Now this view will have no chance left.

In SRT this was always used as we did in (11) dilated just for the unit, so it was larger and not contracted. Even this no one recognized before, but used to tell that time result was viewer in moved IF.

$$
\text { In SRT: } \quad t^{\prime}=\gamma t \rightarrow t^{\prime}>t
$$

The time dilation formula (15) is in each SRT literature for time dilation to find $[3,4,5]$ and it makes the the time $t^{\prime}$ in moved IF longer, more and not viewer as in static IF. How the conclusion was to think then that time was slowed down can be understood only if Einstein was thinking about the one periodic time unit of the clock and the wording in literature is often so like "interval" or "period" or "between two ticks of the clock". Also the wording "dilation" means enlargement, increase, not shorting. So it was the time unit stretched, we agree in that. But then Einstein did not measure the time duration with this stretched unit by adding units one after another while a traveller travels from A to B to get as a variable a viewer scalar number of measured units within a time line as we do now correct in (14) and then to name it also "contraction of time". In German people do not catch this meaning in "dilation" as it is a none German word instead they got learned the result that it is slowing down time. And no one did see that in (15) time was written to get bigger measured in moved IF. But then Einstein told the right result for younger staying twin in moved space shuttle, so at the end he did that measurement too using the stretched time unit to get a lower variable scalar number of stretched units without to write it down. Writing wrong in formula and telling right result. In the length contraction it is both writing and telling same wrong result.

So this is the second logic inconsistency by Einstein's interpretation of Lorentz transformations. He didn't step (14) of measurement the time line willingly but intuitive only.

And by the length he jumped over to the correct result by contracting the distance directly having no units prepared for that measure and concluded that units too have to be contracted. Probably he did take this by his predecessors Poincare and Lorentz, who were authorities of that time.

A well known german youtube blogger, J.Gassner, tells so in his SRT video on youtube "one must put the Lorentz factor so that the moved clocks are slower and lengths are contracted to assure the right results", because it is tricky. A thumb rule.

So, we can conclude that the relativistic stretching the units is a physically relativistic phenomena itself, while a measurement is just the consequence of using that. The unit is one same in all particles being a constant for the given IF and distances are many of an infinite number as they are variables. And they are reciprocally proportional - if one stretches the other shortens. This is a very general relativistic rule for all in nature and therefore is strongly even to look at the measuring the mass too by same rule and the consequence would be that mass hypothetically must be also asymmetrical relativistic attribute as now time and length are both. 
The variable equals the constant unit multiplicate with a variable scalar number to fit in between two locations.

For mass $\boldsymbol{m}$ by relativistic stretching units larger in moved IF

$$
\Delta m^{\prime}=\gamma \Delta m ; \quad \Delta m^{\prime}>\Delta m
$$

and measuring process

$$
m^{\prime}=N \Delta m^{\prime}=N \gamma \Delta m ; \quad m^{\prime}>m
$$

Because $N$ is here number of particles and this stays invariant and that's the difference to length and time, where it is a variable.

The reason for the brain breaker with units and distances is our every day's experience with them. When we write $t=10 \mathrm{sec}$, then in $t$ we feel mathematically as one piece and forget that it is 10 . [sec.] as in all mathematics we don't put values in. Classic physics did no problem, because Lorentz factor is 1 and we use it reciprocally. But in relativity units and measured distances for time and

\begin{tabular}{|c|c|c|c|c|c|}
\hline & \multicolumn{2}{|c|}{ Asymmetric Level Relativity } & \multicolumn{2}{|c|}{ SRT, mixed level } & \\
\hline & stationary IF & moved IF' & stationary IF & moved IF' & \\
\hline time unit & $\Delta t^{\prime}=\gamma \Delta t$ & $\Delta t=\Delta t^{\prime} / \gamma$ & $\Delta t^{\prime}=\gamma \Delta t$ & $\Delta t=\Delta t^{\prime} / \gamma$ & consensus \\
\hline time measured & $t^{\prime}=t / \gamma$ & $t=\gamma t^{\prime}$ & $t^{\prime}=t / \gamma$ & $t=\gamma t^{\prime}$ & consensus \\
\hline length unit & $\Delta l^{\prime}=\gamma \Delta l$ & $\Delta l=\Delta l^{\prime} / \gamma$ & $\Delta l^{\prime}=\Delta / l \gamma$ & $\Delta l=\Delta l^{\prime} / \gamma$ & contradiction \\
\hline length measured & $l^{\prime}=l / \gamma$ & $l=\gamma l^{\prime}$ & $l^{\prime}=l / \gamma$ & $l=l^{\prime} / \gamma$ & contradiction \\
\hline
\end{tabular}
length are treated also reciprocally otherwise it eliminates the whole effect.

Tab. 1. Compare of SRT and present asymmetric Relativity.

In Tab.1 the compare result of two theories is in compact form available.

\section{Time dilation and locations in SRT}

In Fig. 6 is a typical image of the time dilation thought experiment taken from a SRT lexica [2, 3].

We can conclude an important fact, used apparently also by Einstein, that the space points A and A', B and B' are momentary the same in space in both IF. So why then to use them different as in Fig. 2 contacting the space? The points A and B are moved in space transversally and longitudinally too, but when a photon as a localisable particle is meeting at A then it is momentary same point in space for all IF as it is in B too. In this case Einstein used that and we can cite him for that.

In Fig. 6 b) also is clear to understand, that the length of the longer stretched hypotenuse $\mathrm{AB}^{\prime}$ of the triangle is not a result of stretching or contracting the space. So we see in Fig. 6 b) a stretched wave length with respective periodic time also stretched. So where is a contracted space here? The number of wave crests $n$ was invariant the same. This is same model as for transversal Doppler effect.

And they argue in the SRT literature [3] "which expresses the fact, that the moving observers period of the clock $\Delta t$ ' is longer then the period $\Delta t$ in the frame of the clock itself". That's it! They did use the period time of the wave of the photon as an stretched unit calling it "clock". Further they 
consequently did make a frequency as reciprocally of that period time of the clock and did receive the true red lowered frequency. We are discovering a mystified measurement process in details.

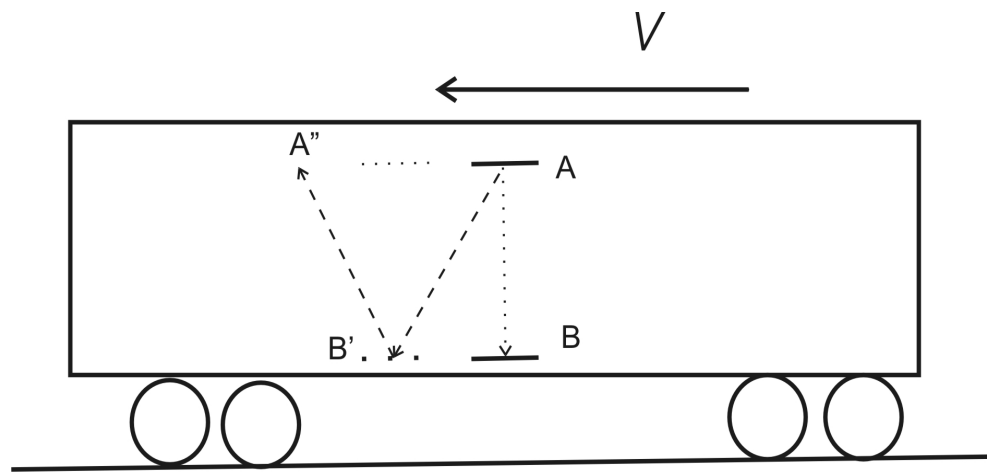

a)

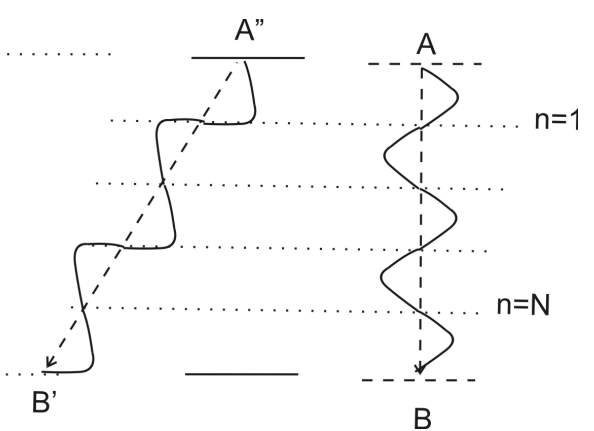

b)

Fig. 6. Transversal light in a moved IF' seen from stationary IF at the rail station a) and a wave stretched transversal in $b$ ).

So it was happening in a time when all was new in relativistic physics and even during 115 years no one of the relativistic anticipates and fans too did find it out. All knowledge about Special Relativity is available in proven and developed didactically 100 years long standard literature available [2, 3 , $4,5]$.

We discovered now that length is asymmetrically too as time is. It was an over interpretation to postulate a symmetry in length contraction with $l^{\prime}=l / \gamma$ and $l=l^{\prime} / \gamma$ at the same time and now it is evident. The new interpreted theory does fit both postulates too, that speed of light is always constant and all physics low is same in all inertial frames.

\section{Elementary particles as elementary Relativistic Measuring Means}

How it happens cinematically has not yet been asked. But we know that all particles with wave properties do have periodic processes, such as wavelength and frequency, and these are suitable measuring devices that every particle always has on board. Our units of length and time are thus composed. Elementary it is precisely these properties that are the actual relativistic surveyors in interactions.

But we know by SRT, that by time dilation all periodical processes are at lower frequency's and atoms emit photons with a red shifted longer wave length. This is the effect of relativistic stretching the particles own on bord relativistic measurement units, which is a consequence of present explained new interpretation of Lorentz transformations and correcting the old SRT. The de Broglie matter wavelength of the atoms emitting such red shifted photons must be also red shifted and stretched in wave length, being a relativistic measurement units of the atoms and particles in it - as every periodical process is to be time dilated.

The relativistic stretching of units is the more elementary primary process at the level of elementary particles in interchange action with time-space. The measurement is then an using of this process. 


\section{Checking invariant constants for relativistically units as a test and discovering a killer paradox for SRT}

We discovered a method of analysing invariant constants, because the units are relativistically to be treated as a test between SRT and the new asymmetric relativity.

Speed of light $c$ does have relativistic units for length and time $[\mathrm{m} / \mathrm{sec}]$, so that means in relativistic dynamic moved IF both relativistic attributes are to be treated each with Lorentz factor according to the theory in question. If we use our present new knowledge that units are to stretch, then we get

$$
c[\gamma m / \gamma s e c]
$$

and eliminating them we get that $c$ is independent of changes by Lorentz factor. That's true.

If we would use the SRT with contracting meter unit meter $/ \gamma$ and stretching time unit as done with time dilation $\gamma \mathrm{sec}$, then we would get erroneous $\left[\right.$ meter $\left./ \gamma^{2} \mathrm{sec}\right]$ and a variable function of light $c\left(1 / \gamma^{2}\right)$ would be none constant.

These effect shows how relativistic units do work in making constants invariant in all IF. They are working hidden eliminating each other. In shadow and invisible we didn't recognise them. But the units stay really stretched.

This is a sudden new argument never expected which contradicts the SRT directly and strongly irreparable. Even if we wouldn't have a working solution it would now be a killer paradox for SRT showing that there is a deep conceptional principle problem.

\section{Decision on twin paradox for a preferred inertial frame with slower time}

Such a decision is not offered by this new relativistic solution either, even if it was the motivation. Rather, "moving lengths become longer" can just as easily be added ad hoc as a phenomena, to accompany the old common thumb rule "moving clocks are slower", which more correct should be "moving time units are longer stretched" as "moving length units are longer stretched" too and therefore measurement get viewer counted units measuring contracted distances and time lines.

We invite the reader to have a look at our other research on relativistics where we solved that problem too and again in a very unexpected conceptional way.

\section{Location authenticity}

We postulated and used this that any particle being at a moment in a location in space and time is in same location for all inertial frames, there cannot be two or more locations at a time for one particle. This is true for all particles included photon and gravitons and is expressed in $\mathrm{A}=\mathrm{A}^{\prime}$. The distances between this locations $\mathrm{A}$ and $\mathrm{B}$ are measured in different IF with different length and time units and are therefore different values. 
The question if an events information will be sooner or later is not of relativity alone. It is classically the same as news always did need time for transport. But all events which happen in one IF also happen in all others. We don't need to break it just to justify the bar-pole paradox which doesn't exist at all. As we do not need any parallel worlds and realities theories too.

We win with demystifying relativism.

\section{Relation to relativistic speed addition}

As far to analyse this in the derivation they where used measured distances and time segments, both used shorted measured values which is correct. No length units failure did distorted that process. Therefore it must fit in new theory too.

\section{Relation to the paradoxes of SRT}

Main goal was that the bar-pole paradox vanished as whole as it is about events and invariance. Other paradoxes like Ehrenfels disc paradox and a rope connecting two rockets paradox never where such ones because this situations cannot be found in nature or it happens as is. The rope in that case will get relaxed and it doesn't mutter at all, if the rope tear apart, then it breaks. That would change nothing in SRT or here in new interpretation too.

\section{Simultaneity}

Being central basic fundament of SRT to avoid the "bar-pole paradox" it has no such importance any more in the present asymmetric relativity. Nevertheless we want to support stronger the invariance of events and a summary event as result of two events must also stay an invariant event. It cannot be prevented in no one of inertial frames just by someone's moving. If it seems so we must change thinking and search for the failure reason. But this is really just opinion of many.

\section{Equality of inertial frames in SRT}

It was too much over interpreted and was to insure that all physics law would be same in all IF. But having allowed time to be prior to that principle was inconsequential.

Now we can see that it is possible to have a level difference between IF and nevertheless all physical laws in same form and same constants are measured in each of IF because they would be measured with relativistically changed units.

So this was something absolute to tell them equal as far as to tell that every one is shorter then the other.

\section{Space in SRT and in new asymmetric level relativity}

Space is stretched and contracted in SRT to serve the relativistic effects under keeping speed of light an invariant constant. This is a philosophical view as consequence of space as a continuum. A 
continuum included the length units and was to stretch and contact in same direction. To think them in opposite direction stretched and contracted was not possible under this fundamental view. This is the deeper reason of Einsteins holding on that not just occasion. The result was that inconsistent using units and measuring method. Einstein must first have decided that space and units are continuum in itself, so units have to be contacted in same direction with space. Then he repaired that with the time dilation alone, inventing the "relativity of the simultaneity" using tricky "synchronized clocks" which was invention of both Poincare and Einstein. The consequence is that "Andromeda paradox" and possibility of different realities which contradicts the invariance of events. This building was build on a wrong philosophical fundament and now it is over.

Subsequently of that space philosophy Einstein stepped over to create the General Relativity Theory GRT and it is very presumably that the same failure is build in in the 5 equations of GRT. If the length units are there inherited contracted with space together instead of stretching, then it is same philosophically deep failure and the result can be similar awful.

Now using the stretched units in order to measure viewer units between space locations $A=A^{\prime}$ and $\mathrm{B}=\mathrm{B}^{\prime}$ we indeed are keeping that space unchanged, static, while we change units only and we got consistency back. The deeper reason is, that those units are length and time dimension units of the elementary particles. How they do it, we can not know for now and we just accept it by phenomena. This another space in that interpretation we didn't invent by some philosophical reason but we looked at the facts in nature which we see in figures here. The locations must stay in same for all IF's.

This is necessary too by the reason that infinite number of particles all have their own IF's and they need to agree about the locations in space. They cannot squeeze the space for themselves each another egoistic version of the reality and change that with their very high frequencies. If one particle was at a moment in a location then it was in that same location $A=A^{\prime}$ in all IF's also. The locations are the same in same space and do not travel. The travelling is the job of the particles only. The question about space expansion we also will recover detailed in another work, but it can be thought for now that the space between $\mathrm{A}$ and $\mathrm{B}$ expands as another phenomena.

\section{Historical review of Dynamic Relativity}

1. Lorentz and Poincare contracted whole matter against an as a fluid meant medium with a certain flow direction and that was wrong; but having changed to "unrecognisable medium" and constant speed of light.

2. Einstein contracted moving matter in an empty space without that fluid, which he also contracted, but contracting the space itself together with the measurement units, which leads to shifting locations physically for each of inertial frames and eliminating the relativistic contraction at all.

3. Now in new interpretation of Lorentz transformations it has been achieved that the measurement units are relativistically stretched, instead of to be contracted for the length alone, in order to measure viewer numerical values for distances and time line segments between spatial locations, which stay in space in same locations and the space itself is not contracted. Elementary particles are entities of the relativistic measurement with own measurement units in them, which interact relativistically with the space and are the source for all macroscopic units. The space is the reason of all and is therefore not empty - at last it contains the still unknown reason. 


\section{Conclusion}

One of the conclusions is that after Lorentz, Poincare and Einstein we have found a third interpretation of the Lorentz transformations. If it is just a possible interpretation or is the only right one we should discover in next coming time by discussing details. On our sides opinion SRT is evidently erroneous by incorrect using the Lorentz transformations on length units and distances due to an erroneous philosophy about space.

Both postulates of Einstein are also covering the new interpretation, which is simpler as we do not need to discuss a complex matter of synchronized clocks and simultaneity to solve a paradox which is avoided now at all. This maybe was the primary misleading idea. Many people will now understand relativity well and probably the reason of all discussion of the past and present was now discovered too. The anticipates of relativity did probably feel intuitively that something was wrong, but no one discovered what in detail.

Now time and length both are relativistically asymmetrical between the Inertial Frames, which makes the relativistic theories more consistent and symmetrical in that unifying principle and it leads to a level relativistic which needs an idea more. We encourage the reader to study our another works on relativity being parallel in publishing process.

References:

[1] Pörtner, L., Muons and time dilation, Bielefeld University, SS 2013, picture on page 14, Available at https://www.physik.uni-bielefeld.de/ yorks/pro13/poertner.pdf

[2] Standard literature of Special Relativity Theory in any edition.

[3] Wikipedia, Time dilation, available at https://en.wikipedia.org/wiki/Time_dilation\#Time_dilation_caused_by_a_relative_velocity

[4] Wikipedia, Length contraction, available at https://en.wikipedia.org/wiki/Length_contraction

[5] Wikipedia, Mass in Special Relativity, available at https://en.wikipedia.org/wiki/Mass_in_special_relativity 
Figures

A

$m$

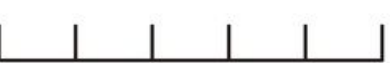

$\mathrm{O}$

a)

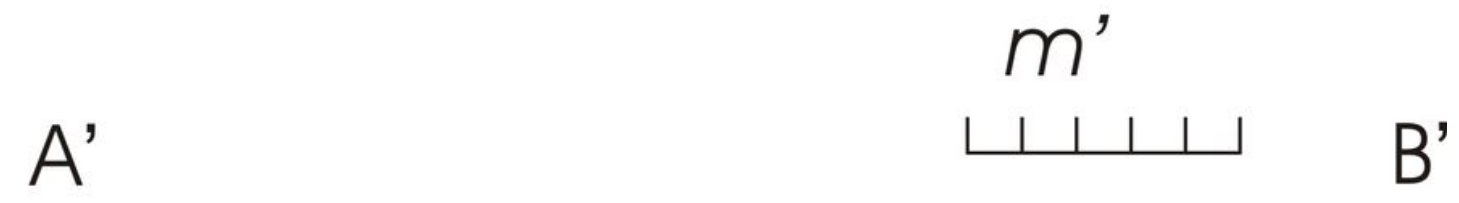

b)

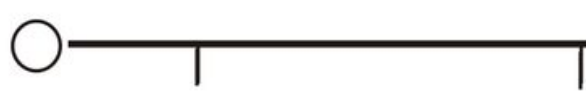

$m^{\prime}$

C)

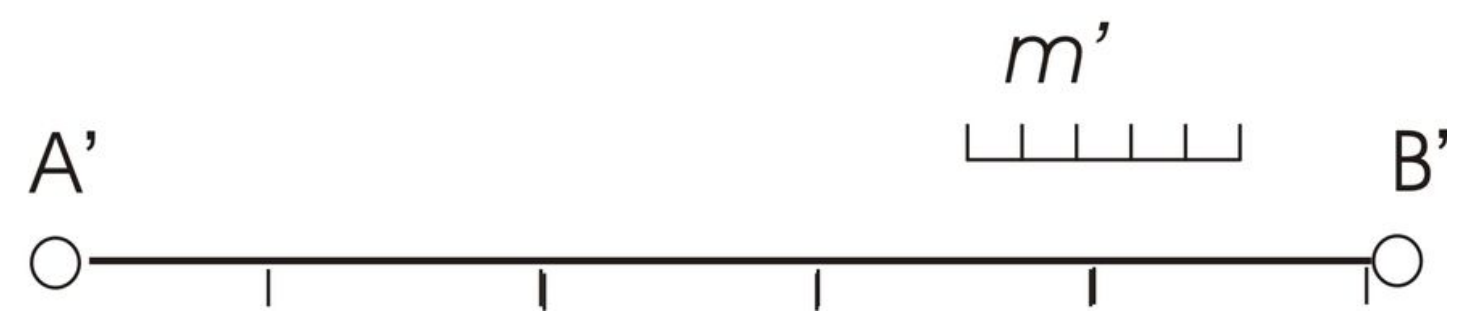

Figure 1

Distance Measurement according to SRT in b), and the avoided step in c). with the failure Muon
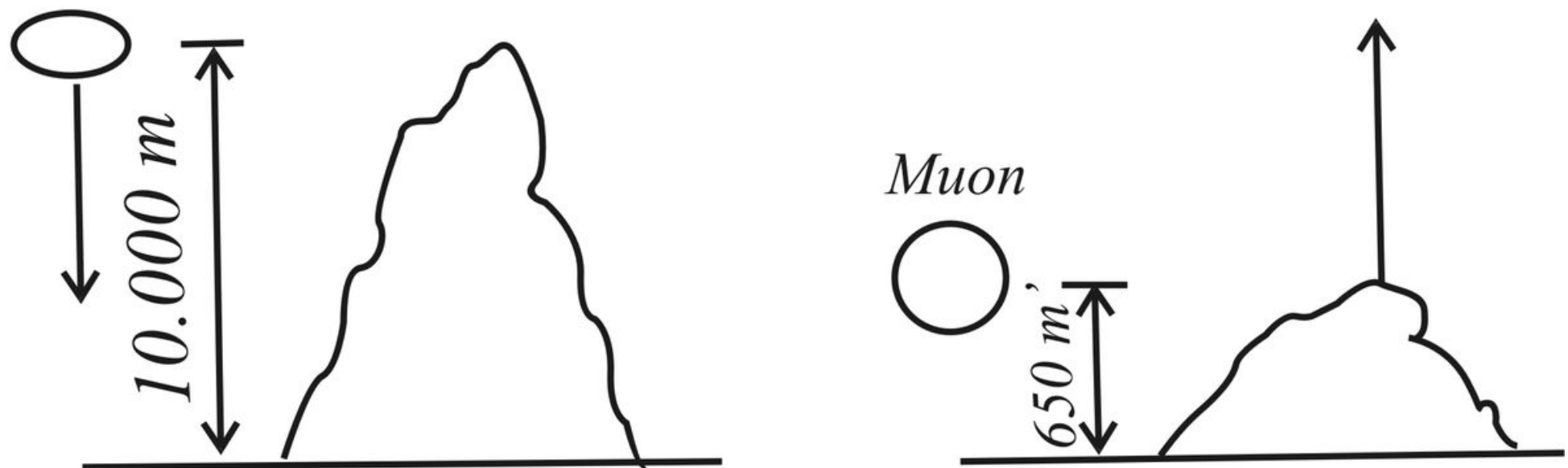
Figure 2

A common representation of the length contraction a Muon track in the atmosphere represented from an university standard teaching materials [1]: a) earths Inertial Frame, b) Muon Inertial Frame.

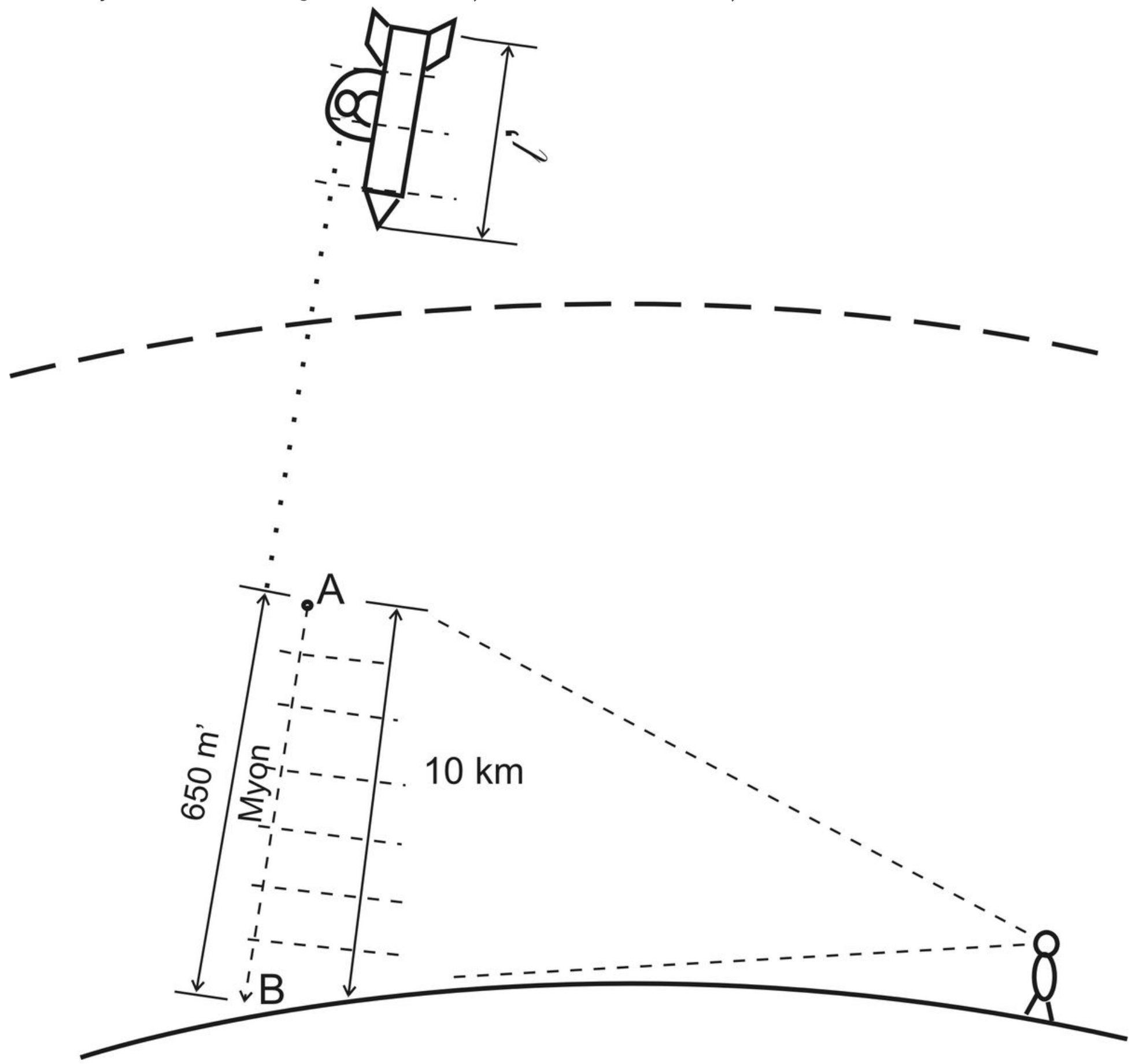

Figure 3

A relativistic Muon observed in the earth's atmosphere from earths Inertial Frame. 
A

$m$

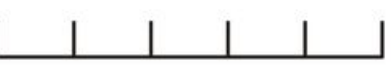

B

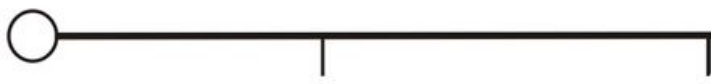

a)

$m^{\prime}$

$A^{\prime}$

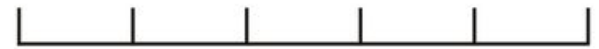

$B^{\prime}$

b)

Figure 4

New measurement method using stretched length unit meter $m^{\prime}=\gamma \mathrm{m}$.

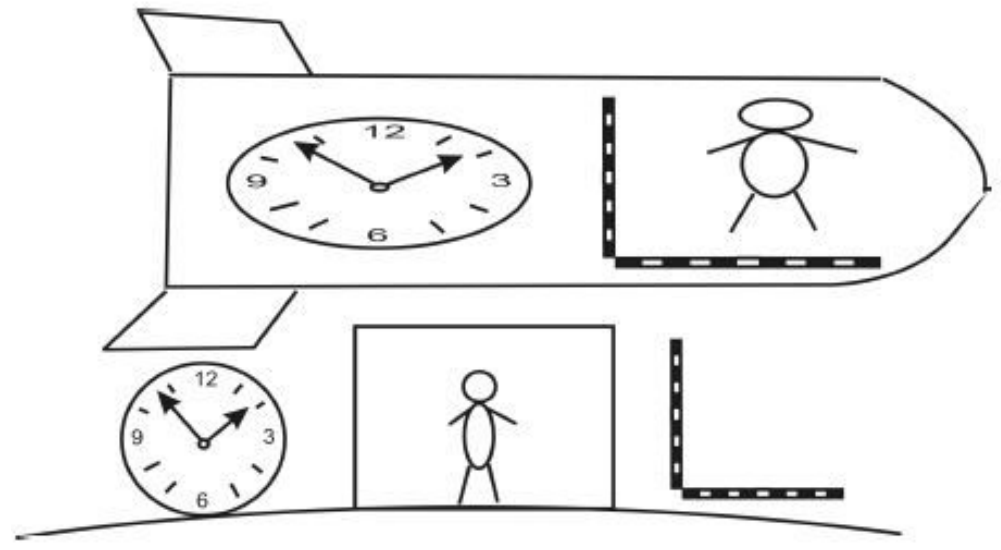

a)

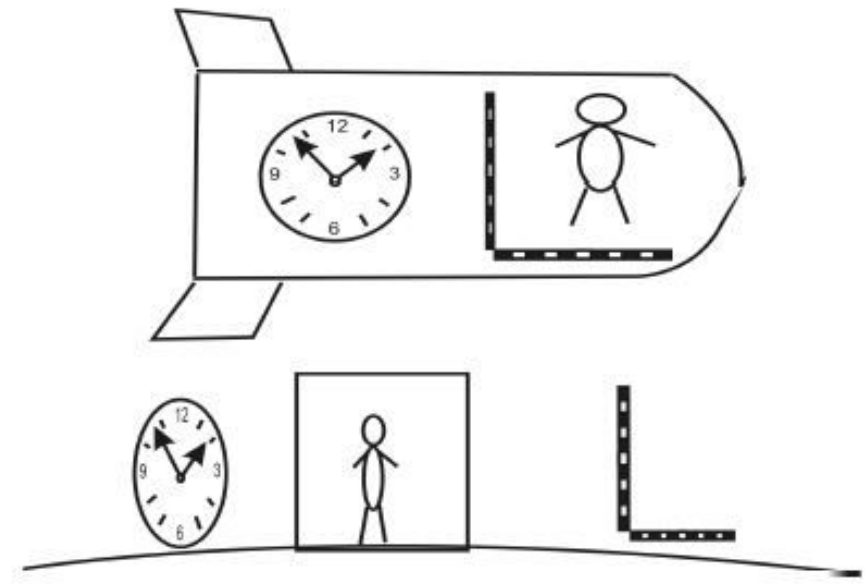

b)

Figure 5 
A new situation free of a "barn-pole paradox" a) in earths IF, b) in space IF both asymmetrically different.

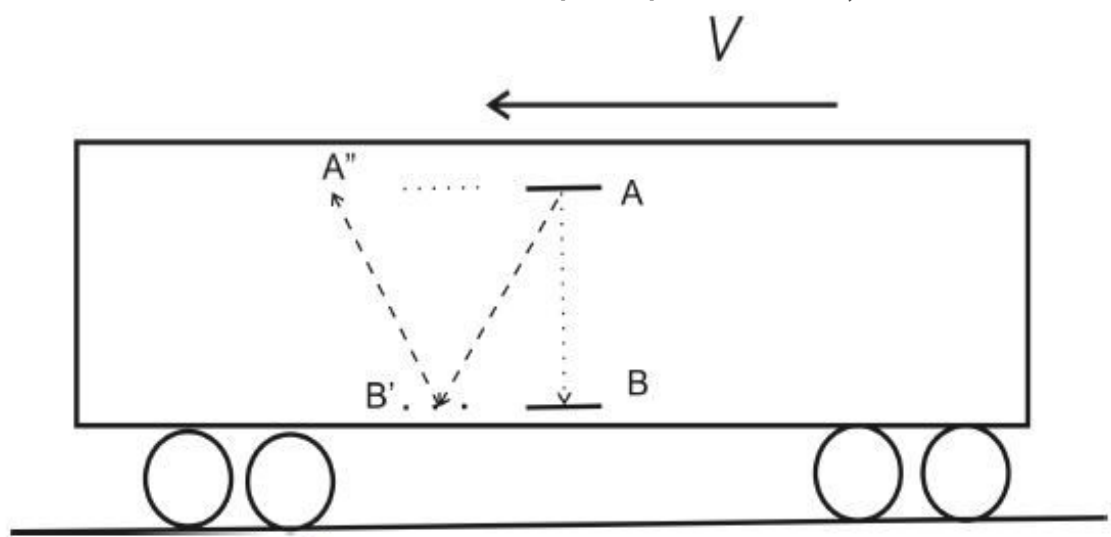

a)

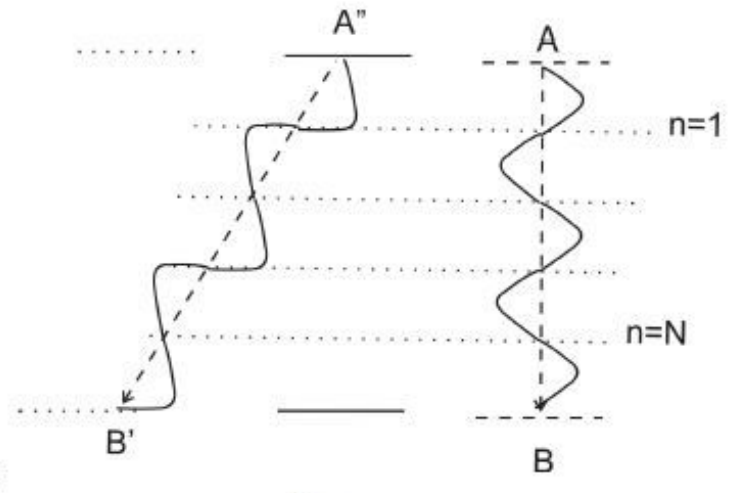

b)

\section{Figure 6}

Transversal light in a moved IF' seen from stationary IF at the rail station a) and a wave stretched transversal in b). 